\title{
Blue-Light-Induced Changes in Arabidopsis Cryptochrome 1 Probed by FTIR Difference Spectroscopy ${ }^{\dagger}$
}

\author{
Tilman Kottke, ${ }^{*, \sharp}$ Alfred Batschauer, ${ }^{\S}$ Margaret Ahmad, ${ }^{\|, \perp}$ and Joachim Heberle ${ }^{\ddagger}, \mathbb{I}$ \\ IBI-2, Structural Biology, Research Center Jülich, 52425 Jülich, Germany, FB Biologie, Philipps-Universität, \\ Karl-von-Frisch-Strasse, 35032 Marburg, Germany, Université Paris VI, Casier 156, 4 Place Jussieu, 75252 Paris Cedex 05, \\ France, Pennsylvania State University, 25 Yearsley Mill Road, Media, Pennsylvania 19063, and Biophysical Chemistry, \\ Universität Bielefeld, Universitätsstrasse 25, 33615 Bielefeld, Germany
}

Received September 28, 2005; Revised Manuscript Received December 15, 2005

\begin{abstract}
Cryptochromes are blue-light photoreceptors that regulate a variety of responses in animals and plants, including circadian entrainment in Drosophila and photomorphogenesis in Arabidopsis. They comprise a photolyase homology region (PHR) of about 500 amino acids and a C-terminal extension of varying length. In the PHR domain, flavin adenine dinucleotide (FAD) is noncovalently bound. The presence of a second chromophore, such as methenyltetrahydrofolate, in animal and plant cryptochromes is still under debate. Arabidopsis cryptochrome 1 (CRY1) has been intensively studied with regard to function and interaction of the protein in vivo and in vitro. However, little is known about the pathway from light absorption to signal transduction on the molecular level. We investigated the full-length CRY1 protein by Fourier transform infrared (FTIR) and UV/vis difference spectroscopy. Starting from the fully oxidized state of the chromophore FAD, a neutral flavoprotein radical is formed upon illumination in the absence of any exogenous electron donor. A preliminary assignment of the chromophore bands is presented. The FTIR difference spectrum reveals only moderate changes in secondary structure of the apoprotein in response to the photoreduction of the chromophore. Deprotonation of an aspartic or glutamic acid, probably D396, accompanies radical formation, as is deduced from the negative band at $1734 \mathrm{~cm}^{-1}$ in $\mathrm{D}_{2} \mathrm{O}$. The main positive band at $1524 \mathrm{~cm}^{-1}$ in the FTIR spectrum shows a strong shift to lower frequencies as compared to other flavoproteins. Together with the unusual blue-shift of the absorption in the visible range to $595 \mathrm{~nm}$, this clearly distinguishes the radical form of CRY1 from those of structurally highly homologous DNA photolyases. As a consequence, the direct comparison of cryptochrome to photolyase in terms of photoreactivity and mechanism has to be made with caution.
\end{abstract}

Cryptochromes are blue-light photoreceptors found in all kingdoms of life (1). Plants use cryptochrome to adapt to their environment via several photomorphogenetic responses. Cryptochrome sets the daily rhythm in both insects (e.g., Drosophila) and plants by regulating the circadian clock. In mammals, including humans, cryptochromes constitute a light-independent integral part of the circadian clock but are possibly also involved in the circadian photoentrainment (2). Cryptochromes contain an N-terminal photolyase homology region (PHR) ${ }^{1}$ of about 500 amino acids (aa). The length of the C-terminal extension varies strongly. In general, planttype cryptochromes contain a longer, species-specific C-

\footnotetext{
This work was supported by the Helmholtz Gemeinschaft VHNG-014 (T.K.), DFG FOR526 (J.H., A.B.), CNRS contrat ACI, and NSF award 0343737 (M.A.).

* Corresponding author. Tel.: +49-2461-61-2036. Fax: +49-246161-2020. E-mail: t.kottke@fz-juelich.de.

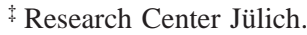

\& Philipps-Universität.

"Université Paris VI.

$\stackrel{\perp}{ }$ Pennsylvania State University.

II Universität Bielefeld.
}

terminal domain (>100 aa) than animal cryptochromes. The $\mathrm{C}$-terminal domain is completely missing in a third class, named cryptochrome DASH (3), which is found mostly in bacteria but also in Arabidopsis (CRY3) (4), Neurospora crassa, and aquatic vertebrates (5). In the PHR domain of all cryptochromes, flavin adenine dinucleotide (FAD) is noncovalently bound as a chromophore $(3,6,7)$. The presence of a second chromophore in cryptochromes is still under debate $(3,8)$. In stoichiometric amounts, 5,10methenyltetrahydrofolate (MTHF) has been shown to associate with members of the cryptochrome DASH family $(5,8,9)$, where it probably acts as a photoantenna $(10)$.

Two plant-type cryptochromes are present in Arabidopsis, CRY1 and CRY2, that regulate light responses such as inhibition of hypocotyl elongation and flowering time,

\footnotetext{
${ }^{1}$ Abbreviations: EDTA, ethylenediaminetetraacetic acid; EPR, electron paramagnetic resonance; FAD, flavin adenine dinucleotide; FTIR, Fourier transform infrared; LOV, light-, oxygen-, and voltagesensitive; MTHF, 5,10-methenyltetrahydrofolate; PHR, photolyase homology region.
} 


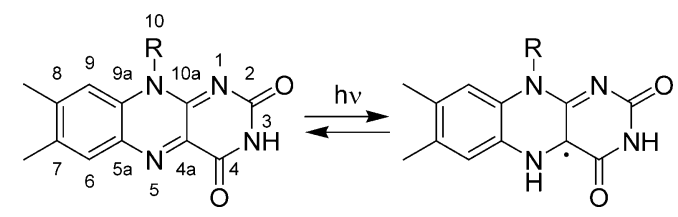

FIGURE 1: Scheme of the conversion from an oxidized flavin to a neutral flavin radical in Arabidopsis cryptochrome 1 upon bluelight illumination.

respectively (11). After heterologous expression in insect cells, the chromophore FAD in CRY1 is present in its oxidized state (7). Action spectra indicate that this is the native redox state of the chromophore (12). There is some evidence from expression in Escherichia coli that MTHF is present as a second chromophore in CRY1 (13). In most preparations, however, CRY1 is isolated devoid of a second chromophore. The structure of the PHR domain of CRY1 devoid of MTHF has been solved by X-ray crystallography to a resolution of $2.6 \AA$, revealing high structural similarity to photolyase (14).

Light induces rapid phosphorylation of CRY1 $(15,16)$ and probably changes its interaction with the E3 ubiquitin ligase COP1 $(17,18)$. Replacement of the N-terminal PHR domain by $\beta$-glucuronidase in transgenic plants leads to a constitutively active phenotype, indicating that the signal is mediated through the C-terminal domain (19). From the influence of light on the rate of proteolytic digestion, it was concluded that a small region of the CRY1 C-terminal domain undergoes conformational changes upon illumination (20). Little is known about the signaling pathway from light absorption to phosphorylation and/or secondary structure changes in CRY1. It has been demonstrated by transient absorption spectroscopy that light induces formation of a neutral flavin radical, which decays on a millisecond time scale (21) (Figure 1). The detection of tryptophan and tyrosine radicals during this process led to a proposal for a photocycle in analogy to photoactivation in photolyases, with the difference that photolyase activation starts from the radical state of the chromophore and results in the formation of reduced FAD (22). In support of this proposal, substitution of two tryptophan residues in CRY1, W324 and W400, resulted in a reduction of light-induced autophosphorylation in vitro and of photoreceptor function in vivo (23).

Vibrational spectroscopy allows one to identify processes taking place at the chromophore, alterations of single amino acid side chains, and conformational changes of the apoprotein with molecular sensitivity. It has been already successfully applied to unravel photoinduced mechanisms in other flavin-containing blue-light receptors, such as phototropin $(24,25)$ and AppA $(26,27)$. In the present study, we investigate for the first time the putative signaling state of the full-length cryptochrome 1 from Arabidopsis by FTIR and UV/vis difference spectroscopy. Spectral changes upon blue-light illumination are unambiguously assigned to the formation of a neutral flavin radical. It is shown that, despite the high structural homology between $E$. coli DNA photolyase and Arabidopsis cryptochrome 1, both the vibrational signature and the visible absorption of the flavin radical differ significantly. The implications of this finding on the mechanism of CRY1 action are discussed.

\section{MATERIALS AND METHODS}

Sample Preparation. Arabidopsis thaliana cryptochrome 1 was isolated from a recombinant baculovirus expression system as reported previously (7). The protein was transferred into $50 \mathrm{mM}$ Tris buffer, $\mathrm{pH} \mathrm{8}$, containing $500 \mathrm{mM} \mathrm{NaCl}$ by repeated ultrafiltration using an Amicon Ultra 4 filter device (Millipore) with $10 \mathrm{kDa}$ cutoff at $4{ }^{\circ} \mathrm{C}$. The final protein concentration was ca. $200 \mu \mathrm{M}$.

A concentrated solution of protein for spectroscopy was obtained by applying a droplet of $5 \mu \mathrm{L}$ to a $\mathrm{CaF}_{2}$ cuvette and reducing the water content under a stream of nitrogen gas. For a hydrated film, the cuvette was subsequently transferred to a homemade anaerobic cell, and the atmosphere was replaced several times by evacuation. The exchange to $\mathrm{D}_{2} \mathrm{O}$ was performed by gentle drying of the protein solution under a stream of nitrogen gas and addition of $5 \mu \mathrm{L}$ of $\mathrm{D}_{2} \mathrm{O}$. This process was repeated four times. Subsequently, the atmosphere was exchanged several times to argon in the anaerobic cell. Cuvettes were sealed by a second $\mathrm{CaF}_{2}$ window without a spacer. The optical path length of the sandwich cuvette was $5-15 \mu \mathrm{m}$.

FTIR and UV/Vis Spectroscopy. FTIR spectroscopy was performed on a Bruker IFS 66v spectrometer with a spectral resolution of $2 \mathrm{~cm}^{-1}$. The temperature of the sample was adjusted to $4{ }^{\circ} \mathrm{C}$ by a circulating water bath. Absorbance spectra were recorded across the whole mid-infrared range. For difference spectroscopy, a broadband interference filter (OCLI) in front of the MCT detector improved the signalto-noise ratio by restricting the recording range to 2000$900 \mathrm{~cm}^{-1}$. Moreover, the filter efficiently blocked stray light. An LED (Luxeon Star, Lumileds) with an emission maximum at $445 \mathrm{~nm}$ (14 $\mathrm{nm}$ full width at half-maximum) provided $40 \mathrm{~mW} / \mathrm{cm}^{2}$ of blue light at the sample. For the concentrated solution and the deuterated film, difference spectra of 1024 scans were recorded under and after illumination with respect to the dark state. For the hydrated film, eight difference spectra of 1024 scans each were averaged after dark periods of $45 \mathrm{~min}$.

UV/vis absorption spectra of the protein films were recorded using a Shimadzu UV 2401 spectrometer with a spectral bandwidth of $2 \mathrm{~nm}$. Temperature and illumination conditions were identical to those in the FTIR experiments. Photoreduction of CRY1 in solution was performed in an anaerobic cuvette in the presence of $10 \mathrm{mM}$ 2-mercaptoethanol and $10 \%$ glycerol after directing a stream of argon onto the solution for $30 \mathrm{~min}$.

\section{RESULTS}

UV/Vis Spectroscopy. The absorption spectrum of cryptochrome 1 from $A$. thaliana was recorded on a hydrated film. It shows two absorption bands centered at 444 and 370 $\mathrm{nm}$, which both exhibit vibrational fine structure (Figure 2A). These are typical features of a flavoprotein housing an oxidized flavin as chromophore. There is no indication for the presence of a second chromophore, such as MTHF, which would strongly absorb with a maximum in the range of 380$410 \mathrm{~nm}$ and an extinction coefficient of $20-25 \mathrm{mM}^{-1} \mathrm{~cm}^{-1}$ (28). For comparison, FAD in water has an extinction coefficient of $11.3 \mathrm{mM}^{-1} \mathrm{~cm}^{-1}$ at $450 \mathrm{~nm}$ (29). Illumination by blue light induced significant changes in the spectrum of CRY1 (Figure 2B). The difference spectra after 120 and 240 


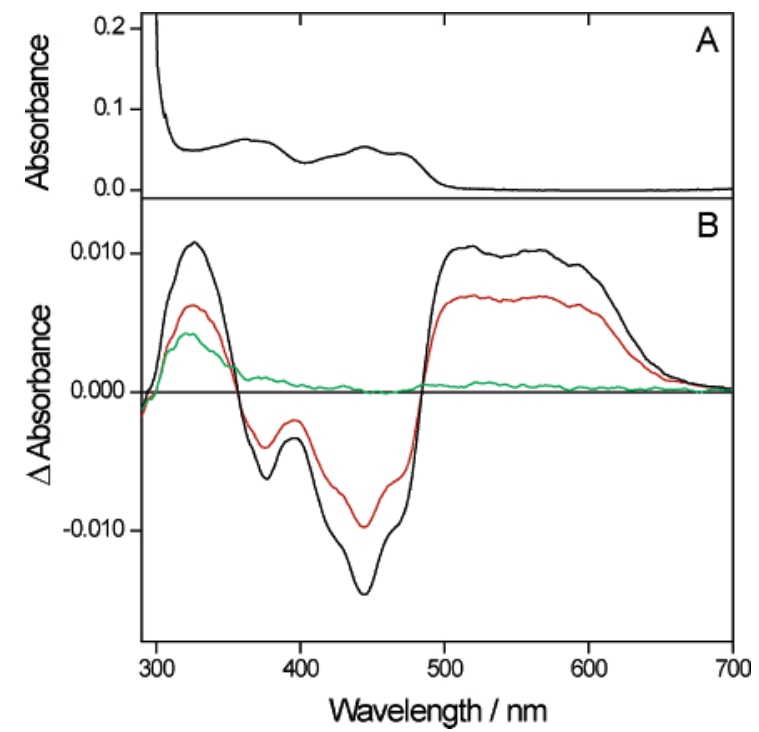

FIGURE 2: (A) UV/vis absorption spectrum of a hydrated film of CRY1 exhibiting the typical features of an oxidized flavoprotein. (B) Sequence of absorption difference spectra recorded after 120 (red) and $240 \mathrm{~s}$ (black) of blue-light illumination, showing the formation of a neutral flavoprotein radical. After 75 min in darkness (green), difference bands of the flavin completely decayed, leaving a residual absorption at $323 \mathrm{~nm}$ and between 490 and $580 \mathrm{~nm}$.

s of illumination exhibit positive bands at 595, 570, 515, and $325 \mathrm{~nm}$. The band positions are in agreement with previous spectra of the neutral flavoprotein radical of CRY1 $(7,21)$. Negative bands at 444 and $375 \mathrm{~nm}$ are caused by the light-induced depletion of the oxidized flavin. Two isosbestic points at 485 and $355 \mathrm{~nm}$ accompany the process, indicating that reduced flavin or side products are not formed in the prolonged photoreaction to the radical.

After $75 \mathrm{~min}$ in the darkness, most difference bands had completely decayed, including all negative bands. The process is therefore fully reversible with respect to the flavin chromophore. In particular, a photoinduced degradation of the FAD was not observed, which would have resulted in a residual negative absorbance at $450 \mathrm{~nm}$ (30). Instead, a positive band at $323 \mathrm{~nm}$ and a weak, broad absorption between 490 and $580 \mathrm{~nm}$ were still present after the decay. The only aromatic compounds in the film besides FAD which might give rise to this absorption are tryptophan, tyrosine, and phenylalanine in their radical state. However, tyrosine radicals show an absorption at $405 \mathrm{~nm}$ (31), and phenylalanine radicals do not absorb at $>450 \mathrm{~nm}$ (32). The spectral features point to the presence of a neutral tryptophan radical, which exhibits two bands at 325 and $510 \mathrm{~nm}$ in aqueous solution (33). It is noted that the ratio of the absorbance at $323 \mathrm{~nm}$ to that at $510 \mathrm{~nm}$ is higher than in the model study in water. It has been proposed for CRY1 that a tryptophan radical is formed by electron transfer to the excited FAD (21). However, this radical was detected as a transient species in a photocycle, decaying within milliseconds into a tyrosine radical. Stable neutral tryptophan radicals are not common in enzymes but have been observed in mutants of Pseudomonas aeruginosa azurin (34) and mouse ribonucleotide reductase (35). To clarify if the species causing the residual absorption is present simultaneously with the flavin radical or if it is formed during the decay of the latter, CRY1 was photoreduced in anaerobic solution in the presence of the exogenous electron donor 2-mercaptoethanol (Figure 3). The

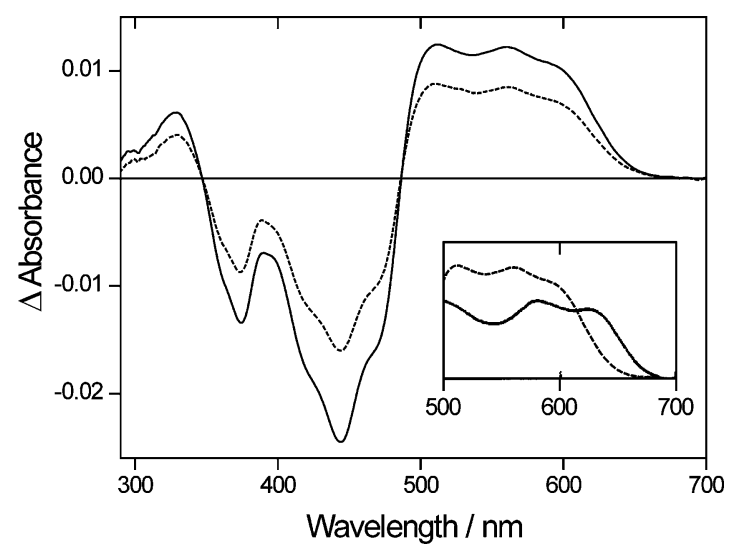

FIGURE 3: Illumination of CRY1 with blue light in anaerobic solution in the presence of the exogenous electron donor 2-mercaptoethanol. Difference spectra were recorded after 20 (dotted line) and $50 \mathrm{~s}$ (solid line) of exposure to light. The inset illustrates the strong blue-shift of the absorption maxima of the CRY1 radical (dashed line) as compared to E. coli DNA photolyase (solid line) (taken from ref 60).

difference spectra differ from those obtained for the film by a significantly reduced absorbance at $325 \mathrm{~nm}$ and a small shift of the isosbestic point from 355 to $347 \mathrm{~nm}$. This result points to a concomitant presence of flavin and tryptophan radical in the film after illumination.

FTIR Difference Spectroscopy. To follow the light-induced processes in CRY1, a concentrated solution was illuminated by blue light for $90 \mathrm{~s}$. Difference spectra with respect to the dark state were recorded under continuous illumination and after illumination but did not differ significantly. In Figure $4 \mathrm{~A}$, the difference spectrum after illumination is presented. The significance of the observed changes is demonstrated by comparison with a difference spectrum without illumination (dotted line). Due to the high water content of the sample, the absorbance in the region $>1550 \mathrm{~cm}^{-1}$ was strongly fluctuating, which prevents any interpretation of vibrational changes in this region. Therefore, a hydrated film of CRY1 was investigated following the same procedure (Figure 4B). As can be seen, the positions of the difference bands at different hydration levels are identical with minor changes in relative intensities. Further drying of the film was not carried out because the protein is only stable in the presence of $500 \mathrm{mM}$ salt and at a high hydration level.

Blue light induced a depletion of the dark state, represented by negative bands, and caused the formation of a new species, characterized by the positive features (Figure 4B). The difference signals did not decay on the time scale of minutes. Most of the negative bands form a pattern that is straightforwardly assigned to vibrational modes of the depleted oxidized flavin chromophore. As a reference, the LOV1 domain of the blue-light receptor Phot from Chlamydomonas reinhardtii was chosen (24). Characteristic bands of flavin at 1578, 1509, 1405, 1349, 1253, 1227, and 1179 $\mathrm{cm}^{-1}$ show shifts of $<5 \mathrm{~cm}^{-1}$ in the direct comparison (Table 1). Only the band at $1543 \mathrm{~cm}^{-1}$ is shifted by $9 \mathrm{~cm}^{-1}$, which will be analyzed in detail in the Discussion section. For FAD in solution, characteristic bands at 1580 and $1548 \mathrm{~cm}^{-1}$ have been reported (36), which is distinctively higher in frequency than in CRY1 (1578 and $1543 \mathrm{~cm}^{-1}$ ). Therefore, significant contributions from free FAD to the difference spectrum of CRY1 can be ruled out. 


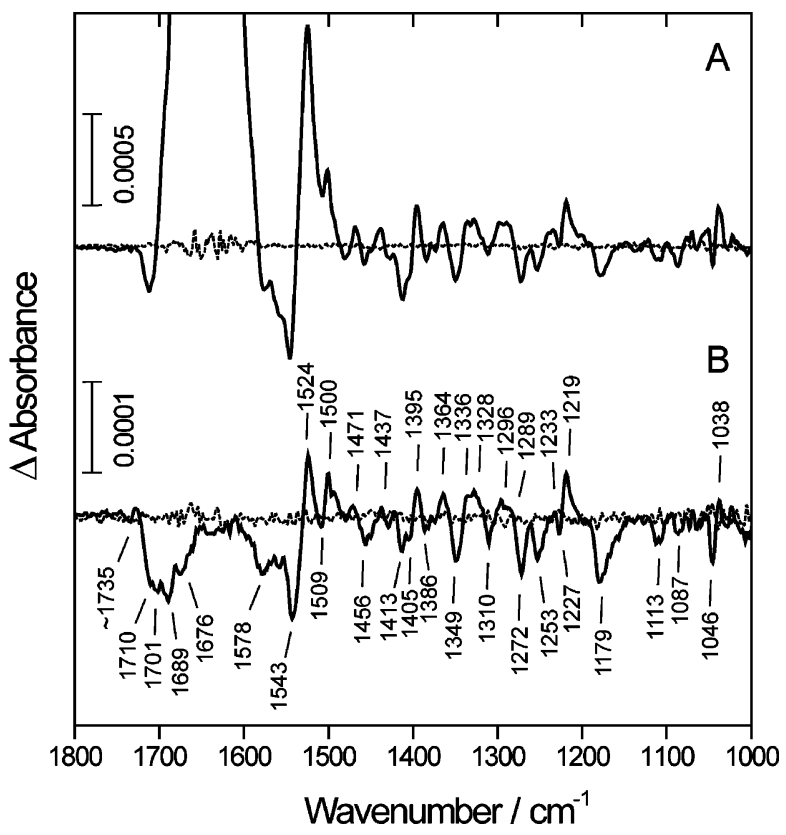

FIGURE 4: FTIR difference spectra of CRY1 with respect to the dark state recorded in the dark (dotted line) and after illumination with blue light (solid line). For comparison, spectra of a concentrated solution (A) and of a hydrated film (B) are shown. Negative bands correspond to the dark state of CRY1, incorporating an oxidized FAD. Positive bands show the light-induced state of CRY1, comprising the vibrational signature of a neutral flavin radical. Bands are obscured by the strong water absorption in the regions $>1550$ (A) and 1660-1590 $\mathrm{cm}^{-1}$ (B), respectively.

Table 1: Negative FTIR Difference Bands for CRY1 after Blue-Light Illumination in Comparison to Those of Flavin in $C$. reinhardtii Phot-LOV1 (24)

\begin{tabular}{cccl}
\hline $\begin{array}{c}\text { A.t. CRY1 } \\
\text { in } \mathrm{H}_{2} \mathrm{O}\end{array}$ & $\begin{array}{c}\text { A.t. } \text { CRY1 } \\
\text { in } \mathrm{D}_{2} \mathrm{O}\end{array}$ & C.r. Phot-LOV1 & \\
\hline 1710 & 1705 & 1712 & $\mathrm{C}_{4}=\mathrm{O}$ \\
& 1638 & 1637 & band I \\
1578 & 1577 & 1583 & band II \\
1543 & 1544 & 1552 & band III \\
1509 & 1512 & 1506 & band IV \\
1405 & 1406 & 1404 & band VI \\
1349 & 1348 & 1352 & band VII \\
1253 & & 1248 & band X \\
1227 & 1229 & 1223 & band XI \\
1179 & 1173 & 1178 & band XII \\
\hline
\end{tabular}

${ }^{a}$ The assignment is based on published data (Ataka et al. (24) and references therein). ${ }^{b}$ The band notation was introduced by Bowman and Spiro (47).

Several positive bands appeared upon illumination, with the most prominent band being located at $1524 \mathrm{~cm}^{-1}$. Together with the bands at $1395,1328,1296$, and $1219 \mathrm{~cm}^{-1}$, this band pattern resembles the resonance Raman spectrum of E. coli DNA photolyase (37), where the flavin chromophore is in the neutral radical state. The shifts between the band positions of the two different proteins are $<5 \mathrm{~cm}^{-1}$. Therefore, the photoproduct of CRY1 can be identified as a neutral flavoprotein radical, in agreement with the conclusion drawn from UV/vis spectroscopy on the same protein film (Figure 2B). The similarity of the band frequencies reflects the fact that the crystal structures of the CRY1 PHR domain from A. thaliana and DNA photolyase from E. coli (38) are very similar, with a root-mean-square deviation of the $\mathrm{C}_{\alpha}$ atoms of only $1.3 \AA$ (14). From the spectral comparison, a
Table 2: Marker Bands of the Neutral Flavin Radical: Comparison of Positive FTIR Difference Bands in CRY1 after Blue-Light Illumination to Resonance Raman Bands of E. coli DNA Photolyase (37)

\begin{tabular}{|c|c|c|c|}
\hline $\begin{array}{l}\text { A.t. } \mathrm{CRY} 1 \\
\text { in } \mathrm{H}_{2} \mathrm{O}\end{array}$ & $\begin{array}{l}\text { A.t. } \mathrm{CRY} 1 \\
\text { in } \mathrm{D}_{2} \mathrm{O}\end{array}$ & $\begin{array}{l}\text { E. coli DNA } \\
\text { photolyase }\end{array}$ & $\begin{array}{l}\text { assignment to } \\
\text { contributions from }{ }^{a}\end{array}$ \\
\hline & 1609 & 1606 & $\begin{array}{c}v(\mathrm{C}(4 \mathrm{a})-\mathrm{C}(10 \mathrm{a}))^{c}+ \\
v(\mathrm{C}(5 \mathrm{a})-\mathrm{C}(9 \mathrm{a}))\end{array}$ \\
\hline & 1596 & $1594^{b}$ & $\begin{array}{l}v(\mathrm{C}-\mathrm{N}(5))+\delta(\mathrm{N}(5)-\mathrm{H})+ \\
v(\mathrm{C}-\mathrm{C})\end{array}$ \\
\hline 1524 & 1525 & 1528 & $v(\mathrm{C}-\mathrm{N}(5))+v(\mathrm{C}-\mathrm{C})$ \\
\hline 1395 & 1393 & 1391 & $v(\mathrm{C}-\mathrm{N}(10))$ \\
\hline 1328 & & 1332 & \\
\hline 1296 & & 1301 & $v(\mathrm{C}-\mathrm{N}(5))$ \\
\hline 1219 & & 1220 & $v(\mathrm{C}-\mathrm{N}(1))+v(\mathrm{C}-\mathrm{N}(3))$ \\
\hline
\end{tabular}

${ }^{a}$ According to Murgida et al. (37) and references therein. ${ }^{b}$ Only visible in $\mathrm{D}_{2} \mathrm{O} .{ }^{c} \mathrm{~A}$ different assignment to a $\mathrm{C}=\mathrm{C}$ stretching mode is discussed by Schelvis et al. (50).

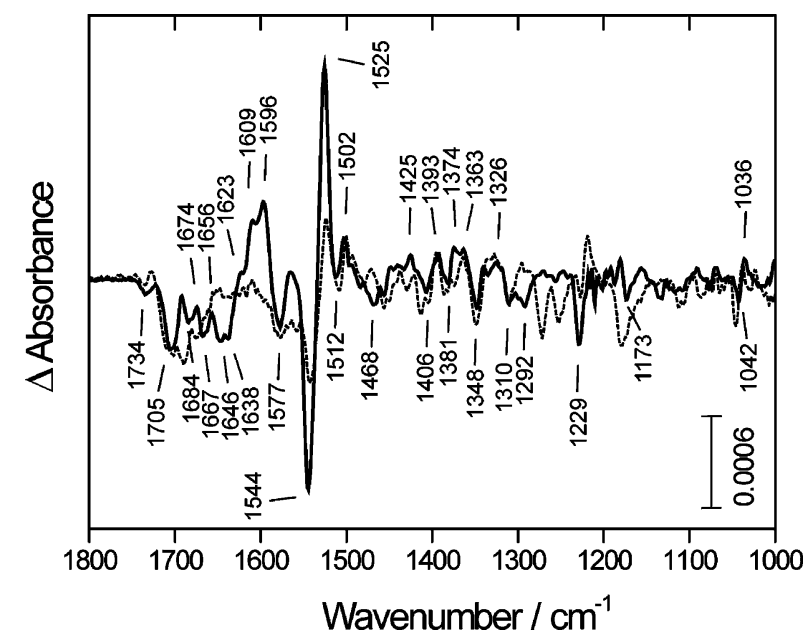

FIGURE 5: FTIR difference spectrum of CRY1 in $\mathrm{D}_{2} \mathrm{O}$ after illumination with blue light. Band frequencies are indicated. The negative band at $1734 \mathrm{~cm}^{-1}$ points to the deprotonation of an aspartic or glutamic acid upon illumination. The difference spectrum of CRY1 in $\mathrm{H}_{2} \mathrm{O}$ (dotted line) is replotted from Figure 4B for comparison

preliminary assignment of the chromophore bands was achieved (Table 2). Many other positive difference bands in the CRY1 spectrum do not have a counterpart in the resonance Raman spectrum of DNA photolyase. They may represent vibrations from the apoprotein that are not enhanced in the resonance Raman approach, which is selective only for the chromophore vibrations.

The light-induced difference spectrum of CRY1 in $\mathrm{D}_{2} \mathrm{O}$ is depicted in Figure 5. The direct comparison with the spectrum in $\mathrm{H}_{2} \mathrm{O}$ shows that the overall shape of the band pattern in the region of $1600-1300 \mathrm{~cm}^{-1}$ did not change much upon deuteration. This agrees with Raman studies on other flavoproteins, which showed that most of the chromophore bands are insensitive to deuteration. This applies to the negative bands of the oxidized flavin (39) as well as to the positive bands of the neutral flavin radical (37) and reflects the fact that the hydrogen at the $\mathrm{N}_{3}$ position is the only exchangeable proton in the isoalloxazine ring. Difference bands are visible in the region of $1660-1590 \mathrm{~cm}^{-1}$ that were obscured in the experiments in $\mathrm{H}_{2} \mathrm{O}$ by the strong water absorption. Some contributions to this region are due to chromophore vibrations: The positive bands at 1596 and $1609 \mathrm{~cm}^{-1}$ can be assigned to flavin modes of the radical 
state by comparison to the Raman spectrum of DNA photolyase in $\mathrm{D}_{2} \mathrm{O}$ (37) (Table 2). The negative band at 1638 $\mathrm{cm}^{-1}$ is assigned to the $\mathrm{C}=\mathrm{C}$ stretching vibration of the benzene ring of flavin, commonly denoted as band I (Table 1). The $\mathrm{C}_{2}=\mathrm{O}$ stretching vibration of oxidized FAD in $\mathrm{D}_{2} \mathrm{O}$ is found at $1656 \mathrm{~cm}^{-1}$ (36) but cannot yet be assigned for CRY1 on the basis of our data. A strong and dominating positive band of a carbonyl vibration of the flavin radical was observed around $1652 \mathrm{~cm}^{-1}$ in studies on riboflavin tetraacetate (40). This band might be overcompensated in CRY1 by a negative amide I band of the protein backbone.

Difference bands in the amide I range of $1695-1615 \mathrm{~cm}^{-1}$ indicate conformational changes of the apoprotein. In CRY1, this spectral region contains several pronounced bands at 1684(-), 1674(+), 1667(-), 1656(+), 1646(-), and 1623(+) $\mathrm{cm}^{-1}$ (Figure 5). The multitude and position of the bands are a strong indication for the occurrence of moderate conformational changes in CRY1 upon illumination. It is noted that some contributions from amino acid side-chain vibrations, such as glutamine or asparagine, cannot be ruled out. Further studies are currently in progress to specifically assign the bands.

A characteristic vibrational band of flavoproteins in the oxidized state is the $\mathrm{C}_{4}=\mathrm{O}$ stretching vibration of the chromophore, which is usually observed at $1723-1687 \mathrm{~cm}^{-1}$ (41). A low frequency of the band implies a strong hydrogen bonding. The crystal structure of the CRY1 PHR domain reveals a single, weak hydrogen bond of $\mathrm{C}_{4}=\mathrm{O}$ to the peptide nitrogen of D392, with a distance of $3.2 \AA$ (14). Therefore, we assign the band at $\sim 1710 \mathrm{~cm}^{-1}$ to the flavin $\mathrm{C}_{4}=\mathrm{O}$ vibration of the CRY1 dark state. The observed weak isotope shift in $\mathrm{D}_{2} \mathrm{O}$ to $1705 \mathrm{~cm}^{-1}$ is in agreement with this assignment, as in other flavoproteins, and for free FAD shifts of $4-13 \mathrm{~cm}^{-1}$ have been determined $(26,36,42)$.

In the hydrated and the deuterated films of CRY1, a negative band is present at the high frequency of $\sim 1735$ and $1734 \mathrm{~cm}^{-1}$, respectively. In this spectral region, only $\mathrm{C}=\mathrm{O}$ stretching modes from carboxylic acids contribute, which implies a deprotonation of an aspartic or glutamic acid upon illumination of cryptochrome 1 . The isotope effect is difficult to quantify, because the position of the band in the hydrated film cannot be precisely determined. The intensity of this signal before averaging over eight measurements is close to the noise level. Shifts of $6-7 \mathrm{~cm}^{-1}$ have been reported in phytochrome and rhodopsin for a similar frequency of the carbonyl mode $(43,44)$. An unusually small shift in CRY1 might be due to a solvent-inaccessible position of the residue.

\section{DISCUSSION}

We have demonstrated that illumination with blue light leads to the formation of a neutral flavoprotein radical in full-length cryptochrome 1 from Arabidopsis. The lightinduced state was characterized using UV/vis and FTIR difference spectroscopy on a well-hydrated film of CRY1. In a previous study using laser flash photolysis, it was concluded that, upon reduction of the flavin by light, a tryptophan radical is formed which decays into a tyrosine radical (21). The recovery of the dark state proceeded with half-lives of 1,5 , and $>100 \mathrm{~ms}$. In the protein film, an accumulation of the flavin radical state with the unresolved half-life of $>100 \mathrm{~ms}$ might have occurred under continuous illumination, as it cannot be deduced from the time-resolved data that the process is completely reversible. During the accumulation and after the complete decay of the flavin radical in the film, an additional species is detected, the spectral properties of which resemble those of a tryptophan radical (Figures 2B and 3). This might be interpreted as the stabilization of an intermediate state in the proposed CRY1 photocycle under our experimental conditions. The discrepancy in the radical stabilities might be caused by exposing the concentrated protein in a closed compartment to high light intensity. The long illumination times were necessary for the concomitant FTIR measurements to drive sufficient molecules into the light-induced state. This is mandatory to obtain a reasonable signal-to-noise ratio, taking into account the large size of the protein of $75 \mathrm{kDa}$ and the low quantum yield of $\sim 2 \%$ of the photoreduction to the radical in CRY1 (21). In contrast to previous studies $(7,21)$, no exogenous electron donor such as ethylenediaminetetraacetic acid (EDTA) or 2-mercaptoethanol was added to stabilize the radical. Furthermore, the presence of oxygen was not thoroughly excluded during preparation. This clearly distinguishes our procedure from the common anaerobic photoreduction of most flavoproteins to the anionic or neutral radical. Only in the LOV2-C450A mutant of the blue-light receptor phototropin, addition of an exogenous donor was not necessary for formation of the radical (45). The authors used this finding to propose a radical mechanism for LOV domain photoreactions in general. We analogously conclude from our observations that the demonstrated photoreaction of CRY1 is an intrinsic property of the protein.

Formation of the neutral flavin radical in CRY1 requires protonation of the flavin at $\mathrm{N}_{5}$. The negative band at 1734 $\mathrm{cm}^{-1}$ in the CRY1 difference spectrum in $\mathrm{D}_{2} \mathrm{O}$ points to the deprotonation of a glutamic or aspartic acid upon radical formation. Accordingly, four candidates for the role of a proton donor are located in the vicinity of the flavin: D392, D359, D390, and D396. The former two are not sufficiently close to the flavin $\mathrm{N}_{5}$, with a distance to the side-chain oxygen of 7.2 and $7.1 \AA$, respectively (Figure 6). D390 forms a conserved salt bridge with R362, parallel to the isoalloxazine ring (38). A proton release from this site cannot cause the observed signal, as D390 is present in its deprotonated form. Therefore, we consider D396 to be the most likely candidate, with a distance of $3.8 \AA$ to flavin $\mathrm{N}_{5}$ (Figure 6). This interpretation is supported by the fact that the corresponding amino acid in E. coli DNA photolyase is an asparagine (N378), which stabilizes the protonated radical by a hydrogen bond to $\mathrm{N}_{5}(38)$. Moreover, D396 is located between FAD and W400 (Figure 6), which has been shown to be an essential residue for radical formation in CRY1 (23). W400 might act as the primary electron donor in a sequence of three tryptophans (W400, W377, and W324), in analogy with the electron-transfer pathway for photoreduction in $E$. coli photolyase (22). Accordingly, the negative band at 1734 $\mathrm{cm}^{-1}$ in the CRY1 difference spectrum in $\mathrm{D}_{2} \mathrm{O}$ might be assigned to the $\mathrm{C}=\mathrm{O}$ stretching vibration of protonated D396 in the initial state (Figure 5). The presence of a protonated D396 in the dark requires an apolar environment, which is provided by M381, F384, W385, D392, S395, A397, and W400 in a $5 \AA$ shell around D396. We propose that proton transfer from D396 to the flavin $\mathrm{N}_{5}$ represents a crucial step in the photoactivation mechanism of CRY1. 


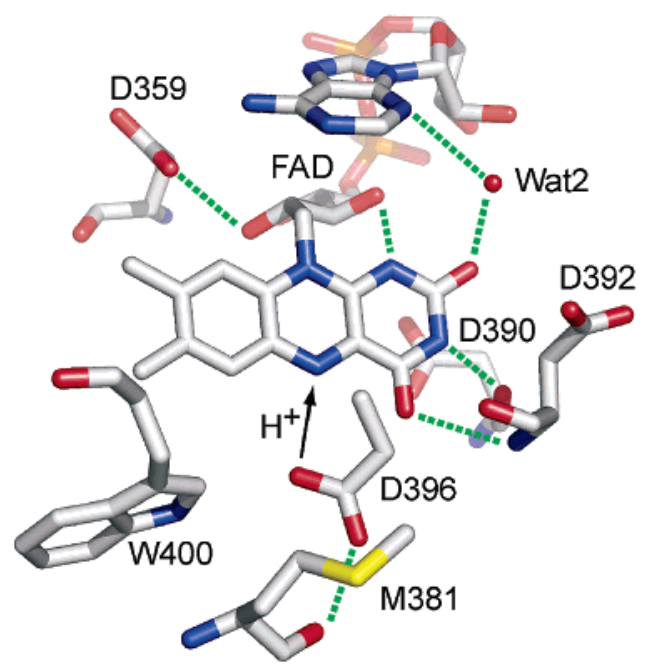

FIGURE 6: Chromophore pocket of Arabidopsis CRY1 obtained from the crystal structure of the PHR domain (14). Possible hydrogen bonds are depicted as green dotted lines. Upon illumination, D396 is proposed to donate the proton to the $\mathrm{N}_{5}$-position of the oxidized flavin for formation of the neutral flavin radical, whereas W400 might act as the electron donor. Two other putative proton donors, D359 and D392, can be excluded due to their distance from flavin $\mathrm{N}_{5}$. D390 is involved in a salt bridge in its deprotonated form.

The position of the major negative band at $1543 \mathrm{~cm}^{-1}$ is shifted to lower frequencies as compared to other flavoproteins and flavoprotein radicals (Figure 4B). Oxidized flavoproteins with a planar isoalloxazine ring, such as CRY1, generally show bands in the range of $1553-1544 \mathrm{~cm}^{-1}$ (46). This band has been assigned to the diazabuta-1,3-diene moiety of flavin $\left(\mathrm{N}_{5}=\mathrm{C}_{4 \mathrm{a}}-\mathrm{C}_{10 \mathrm{a}}=\mathrm{N}_{1}\right)$, with a strong contribution from the $\mathrm{C}_{4 \mathrm{a}}-\mathrm{C}_{10 \mathrm{a}}$ mode $(46,47)$. The low frequency of $1543(-) \mathrm{cm}^{-1}$ can be attributed to the lack of hydrogen bonding to the $\mathrm{N}_{1}$ and $\mathrm{N}_{5}$ positions of the oxidized flavin (46), which leads to a weakening of the $\mathrm{C}_{4 \mathrm{a}}-\mathrm{C}_{10 \mathrm{a}}$ bond. This is in line with the crystal structure data of the PHR domain of CRY1 (14), where the only donor within H-bonding distance to one of these nitrogen atoms is the $3^{\prime}$ hydroxyl group of the riboflavin moiety.

The most prominent positive band in the difference spectrum is located at $1524 \mathrm{~cm}^{-1}$, which is to our knowledge the lowest frequency of this radical mode determined in any flavoprotein so far. For flavoprotein radicals, frequencies of 1539-1532 $\mathrm{cm}^{-1}$ have been determined, with photolyase showing an exceptionally low frequency of $1528 \mathrm{~cm}^{-1}$ (37). Recent FTIR experiments on DNA photolyase show a higher position of the band of $1532 \mathrm{~cm}^{-1}$ (48). Therefore, the band at $1524(+) \mathrm{cm}^{-1}$ of the CRY1 radical is downshifted by $4-8$ $\mathrm{cm}^{-1}$ in comparison to the spectrum of DNA photolyase, despite the highly similar structure. This mode was analyzed to include distinct contributions from $\mathrm{N}_{5}-\mathrm{C}$ stretching vibrations, which was concluded from a strong downshift to $1516 \mathrm{~cm}^{-1}$ for the $\mathrm{N}_{5}$-methylated radical in water $(37$, 49). The weakening of these $\mathrm{N}_{5}-\mathrm{C}$ bonds may originate from the highly localized spin density on these atoms, as has been proposed for DNA photolyase (50) from electron paramagnetic resonance (EPR) experiments (51). The particularity of the electron density in the FAD radical in CRY1 is also reflected in the UV/vis difference spectra (Figures 2 and 3). The shoulder at $595 \mathrm{~nm}$ of the lowest energy transition is blue-shifted by $30 \mathrm{~nm}$ as compared to DNA photolyase (52)
(Figure 3 inset) and is not typical for flavoprotein radicals, which absorb at $\lambda>600 \mathrm{~nm}$ (53). Studies on a 5-ethylated flavin radical revealed a blue-shift of the absorption upon changing the solvent from benzene over ethanol to water (53). Additionally, the shorter wavelength maximum of the doublet around $600 \mathrm{~nm}$ was getting more pronounced, which is also observed in the comparison of CRY1 to photolyase (Figure 3 inset). Therefore, the differences might be partly explained by a higher polarity of the binding pocket in CRY1 than in photolyase. Two asparagines close to the chromophore in E. coli DNA photolyase (D341 and D378) are replaced by aspartates or aspartic acids in CRY1 (D359 and D396). However, stronger molecular contributions to the spectral shift have yet to be revealed. Taken together, these results demonstrate the uniqueness of the spectroscopic properties of the CRY1 radical.

It has been claimed that the reduced state of flavin is the native state of cryptochromes in vivo (10). Moreover, oxidized and radical states were proposed to be artifacts from the preparation procedure (10), as has been shown for photolyase. This might be true for members of the cryptochrome DASH family, such as cryptochrome 1 from Vibrio cholerae. However, the action spectrum of hypocotyl growth inhibition mediated by Arabidopsis CRY1 clearly deviates from that of photolyase-mediated DNA repair. In the former case, the maximum activity was found at 390-480 nm (12), as opposed to 320-440 nm for E. coli DNA photolyase (54). For photolyase, the action spectrum matches the absorption spectrum of the enzyme, which is dominated by the contribution of the second chromophore MTHF. The reduced flavin present in photolyase does not absorb significantly in this spectral range. For CRY1, the action spectrum provides evidence for the presence of an oxidized flavin without strong contributions from MTHF. It resembles the absorption spectrum of CRY1 obtained in vitro after purification from insect cells (Figure 2A). It is noted that the absorption spectrum taken directly after cell lyses shows a single maximum at $420 \mathrm{~nm}$ (12), probably due to the presence of MTHF (13), and therefore deviates from the action spectrum. This discrepancy indicates that, in contrast to photolyases, the antenna pigment does not contribute significantly to the action spectrum in CRY1. This might be caused by unspecific binding and, consequently, unfavorable orientation of MTHF relative to flavin. The comparison to DNA photolyase shows that the binding pocket for MTHF is largely occupied with amino acid side chains in CRY1 (14) and that five out of six residues involved in binding of MTHF are not conserved in CRY1 (38). Thus, it is unlikely that the absence of MTHF in our preparation as well as in the previous work (21) fundamentally changed the light-dependent behavior of CRY1. From the present study, we conclude that photolyase and cryptochrome radical differ significantly in the electron distribution in the isoalloxazine ring. This complicates a direct comparison of cryptochrome with the photolyase system in terms of photoreactivity and mechanism. It is therefore not unlikely that the functional mechanism of CRY1 indeed starts from the oxidized state of FAD.

In a current model for CRY1 signal transduction, it is proposed that a light-induced conformational change of the protein effects the interaction of CRY1 with the binding partners COP1 and HY5, resulting in a degradation of the transcriptional regulator HY5 (55). From our FTIR data, only 
moderate conformational changes accompany the formation of the radical (Figure 5). This is in line with a proteolytic digestion assay on CRY1, where it was concluded that only a relatively small region of the C-terminal extension rearranges in response to light (20). The extent of conformational change does not necessarily need to be dramatic, since the interaction of CRY1 with COP1 is only altered, not lost. Moreover, the biggest part of the C-terminal extension was predicted to be unstructured (20), in which case changes in conformation are difficult to detect by FTIR spectroscopy. Inside the PHR domain, the signal might be transferred from the chromophore to the C-terminal extension without strong structural disturbances. In the case of phototropin, it has been shown by X-ray crystallography that light-induced changes in conformation in the LOV domains themselves are small $(56,57)$, whereas the activation of the kinase proceeds via unfolding of an $\alpha$-helix downstream of $\operatorname{LOV} 2(58,59)$.

\section{CONCLUSIONS}

Arabidopsis CRY1 is currently the best model system available for studying processes of cryptochrome signal transduction. The function of the protein and its interaction with signaling partners have been intensively studied. The availability of time-resolved absorption spectra on the fulllength protein as well as a crystal structure of the PHR domain facilitates interpretation of changes at the molecular level. On this basis, we were able to characterize the vibrational changes occurring in the full-length CRY1 upon illumination with blue light. The reversible formation of a neutral flavin radical by blue light is accompanied by changes in secondary structure of the apoprotein. Proton transfer from an aspartic or glutamic acid, probably D396, to the flavin is proposed to represent an important step in the photoactivation of CRY1. Major negative and positive difference bands were assigned by comparison to those of other flavoproteins. This analysis is novel for FTIR studies on flavoprotein radicals in general and will facilitate future work on the cryptochrome signaling process.

\section{ACKNOWLEDGMENT}

We thank Georg Büldt (Forschungszentrum Jülich) for generous support.

\section{SUPPORTING INFORMATION AVAILABLE}

Infrared absorbance spectra of CRY1 showing the hydration level of the protein films and the exchange ratio to $\mathrm{D}_{2} \mathrm{O}$. This material is available free of charge via the Internet at http://pubs.acs.org.

\section{REFERENCES}

1. Cashmore, A. R., Jarillo, J. A., Wu, Y. J., and Liu, D. (1999) Cryptochromes: blue light receptors for plants and animals, Science 284, 760-765.

2. Partch, C. L., and Sancar, A. (2005) Cryptochromes and circadian photoreception in animals, Methods Enzymol. 393, 726-745.

3. Brudler, R., Hitomi, K., Daiyasu, H., Toh, H., Kucho, K., Ishiura, M., Kanehisa, M., Roberts, V. A., Todo, T., Tainer, J. A., and Getzoff, E. D. (2003) Identification of a new cryptochrome class. Structure, function, and evolution, Mol. Cell. 11, 59-67.

4. Kleine, T., Lockhart, P., and Batschauer, A. (2003) An Arabidopsis protein closely related to Synechocystis cryptochrome is targeted to organelles, Plant J. 35, 93-103.
5. Daiyasu, H., Ishikawa, T., Kuma, K., Iwai, S., Todo, T., and Toh, H. (2004) Identification of cryptochrome DASH from vertebrates, Genes Cells 9, 479-495.

6. Hsu, D. S., Zhao, X., Zhao, S., Kazantsev, A., Wang, R. P., Todo, T., Wei, Y. F., and Sancar, A. (1996) Putative human blue-light photoreceptors hCRY1 and hCRY2 are flavoproteins, Biochemistry 35, 13871-13877.

7. Lin, C., Robertson, D. E., Ahmad, M., Raibekas, A. A., Jorns, M. S., Dutton, P. L., and Cashmore, A. R. (1995) Association of flavin adenine dinucleotide with the Arabidopsis blue light receptor CRY1, Science 269, 968-970.

8. Worthington, E. N., Kavakli, I. H., Berrocal-Tito, G., Bondo, B. E., and Sancar, A. (2003) Purification and characterization of three members of the photolyase/cryptochrome family blue-light photoreceptors from Vibrio cholerae, J. Biol. Chem. 278, 3914339154.

9. Pokorny, R., Klar, T., Essen, L. O., and Batschauer, A. (2005) Crystallization and preliminary X-ray analysis of cryptochrome 3 from Arabidopsis thaliana, Acta Crystallogr. F61, 935-938.

10. Saxena, C., Wang, H., Kavakli, I. H., Sancar, A., and Zhong, D. (2005) Ultrafast dynamics of resonance energy transfer in cryptochrome, J. Am. Chem. Soc. 127, 7984-7985.

11. Lin, C., and Shalitin, D. (2003) Cryptochrome structure and signal transduction, Аnпи. Rev. Plant Biol. 54, 469-496.

12. Ahmad, M., Grancher, N., Heil, M., Black, R. C., Giovani, B., Galland, P., and Lardemer, D. (2002) Action spectrum for cryptochrome-dependent hypocotyl growth inhibition in Arabidopsis, Plant Physiol. 129, 774-785.

13. Malhotra, K., Kim, S. T., Batschauer, A., Dawut, L., and Sancar, A. (1995) Putative blue-light photoreceptors from Arabidopsis thaliana and Sinapis alba with a high degree of sequence homology to DNA photolyase contain the two photolyase cofactors but lack DNA repair activity, Biochemistry 34, 6892-6899.

14. Brautigam, C. A., Smith, B. S., Ma, Z., Palnitkar, M., Tomchick, D. R., Machius, M., and Deisenhofer, J. (2004) Structure of the photolyase-like domain of cryptochrome 1 from Arabidopsis thaliana, Proc. Natl. Acad. Sci. U.S.A. 101, 12142-12147.

15. Shalitin, D., Yu, X., Maymon, M., Mockler, T., and Lin, C. (2003) Blue light-dependent in vivo and in vitro phosphorylation of Arabidopsis cryptochrome 1, Plant Cell 15, 2421-2429.

16. Bouly, J. P., Giovani, B., Djamei, A., Mueller, M., Zeugner, A., Dudkin, E. A., Batschauer, A., and Ahmad, M. (2003) Novel ATPbinding and autophosphorylation activity associated with Arabidopsis and human cryptochrome-1, Eur. J. Biochem. 270, 29212928.

17. Wang, H., Ma, L. G., Li, J. M., Zhao, H. Y., and Deng, X. W. (2001) Direct interaction of Arabidopsis cryptochromes with COP1 in light control development, Science 294, 154-158.

18. Yang, H. Q., Tang, R. H., and Cashmore, A. R. (2001) The signaling mechanism of Arabidopsis CRY1 involves direct interaction with COP1, Plant Cell 13, 2573-2587.

19. Yang, H. Q., Wu, Y. J., Tang, R. H., Liu, D., Liu, Y., and Cashmore, A. R. (2000) The C termini of Arabidopsis cryptochromes mediate a constitutive light response, Cell 103, 815827.

20. Partch, C. L., Clarkson, M. W., Ozgur, S., Lee, A. L., and Sancar, A. (2005) Role of structural plasticity in signal transduction by the cryptochrome blue-light photoreceptor, Biochemistry 44, 3795-3805.

21. Giovani, B., Byrdin, M., Ahmad, M., and Brettel, K. (2003) Lightinduced electron transfer in a cryptochrome blue-light photoreceptor, Nat. Struct. Biol. 10, 489-490.

22. Aubert, C., Vos, M. H., Mathis, P., Eker, A. P., and Brettel, K. (2000) Intraprotein radical transfer during photoactivation of DNA photolyase, Nature 405, 586-590.

23. Zeugner, A., Byrdin, M., Bouly, J. P., Bakrim, N., Giovani, B., Brettel, K., and Ahmad, M. (2005) Light-induced Electron Transfer in Arabidopsis Cryptochrome-1 Correlates with in Vivo Function, J. Biol. Chem. 280, 19437-19440.

24. Ataka, K., Hegemann, P., and Heberle, J. (2003) Vibrational Spectroscopy of an Algal Phot-LOV1 Domain Probes the Molecular Changes Associated with Blue-Light Reception, Biophys. J. $84,466-474$.

25. Swartz, T. E., Wenzel, P. J., Corchnoy, S. B., Briggs, W. R., and Bogomolni, R. A. (2002) Vibration Spectroscopy Reveals LightInduced Chromophore and Protein Structural Changes in the LOV2 Domain of the Plant Blue-Light Receptor Phototropin 1, Biochemistry 41, 7183-7189. 
26. Masuda, S., Hasegawa, K., and Ono, T. A. (2005) Light-induced structural changes of apoprotein and chromophore in the sensor of blue light using FAD (BLUF) domain of AppA for a signaling state, Biochemistry 44, 1215-1224.

27. Laan, W., Bednarz, T., Heberle, J., and Hellingwerf, K. J. (2004) Chromophore composition of a heterologously expressed BLUFdomain, Photochem. Photobiol. Sci. 3, 1011-1016.

28. Malhotra, K., Kim, S. T., and Sancar, A. (1994) Characterization of a medium wavelength type DNA photolyase: purification and properties of photolyase from Bacillus firmus, Biochemistry 33, 8712-8718.

29. Siegel, L. M. (1978) Quantitative determination of noncovalently bound flavins: types and methods of analysis, Methods Enzymol. $53,419-429$.

30. Holzer, W., Shirdel, J., Zirak, P., Penzkofer, A., Hegemann, P., Deutzmann, R., and Hochmuth, E. (2005) Photo-induced degradation of some flavins in aqueous solution, Chem. Phys. 308, 6978.

31. Bent, D. V., and Hayon, E. (1975) Excited-state chemistry of aromatic amino acids and related peptides. I. Tyrosine, J. Am. Chem. Soc. 97, 2599-2606.

32. Bent, D. V., and Hayon, E. (1975) Excited-state chemistry of aromatic amino acids and related peptides. II. Phenylalanine, $J$. Am. Chem. Soc. 97, 2606-2612.

33. Solar, S., Getoff, N., Surdhar, P. S., Armstrong, D. A., and Singh, A. (1991) Oxidation of Tryptophan and $N$-Methylindole by N3., Br2-, and (Scn)2- Radicals in Light-Water and Heavy-Water Solutions-A Pulse-Radiolysis Study, J. Phys. Chem. 95, 36393643.

34. Potsch, S., Lendzian, F., Ingemarson, R., Hornberg, A., Thelander, L., Lubitz, W., Lassmann, G., and Graslund, A. (1999) The ironoxygen reconstitution reaction in protein R2-Tyr-177 mutants of mouse ribonucleotide reductase. Epr and electron nuclear double resonance studies on a new transient tryptophan radical, J. Biol. Chem. 274, 17696-17704.

35. Miller, J. E., Gradinaru, C., Crane, B. R., Di Bilio, A. J., Wehbi, W. A., Un, S., Winkler, J. R., and Gray, H. B. (2003) Spectroscopy and reactivity of a photogenerated tryptophan radical in a structurally defined protein environment, J. Am. Chem. Soc. 125, $14220-14221$.

36. Wille, G., Ritter, M., Friedemann, R., Mäntele, W., and Hübner, G. (2003) Redox-triggered FTIR difference spectra of FAD in aqueous solution and bound to flavoproteins, Biochemistry 42, 14814-14821.

37. Murgida, D. H., Schleicher, E., Bacher, A., Richter, G., and Hildebrandt, P. (2001) Resonance Raman spectroscopic study of the neutral flavin radical complex of DNA photolyase from Escherichia coli, J. Raman Spectrosc. 32, 551-556.

38. Park, H. W., Kim, S. T., Sancar, A., and Deisenhofer, J. (1995) Crystal structure of DNA photolyase from Escherichia coli, Science 268, 1866-1872.

39. Visser, A. J., Vervoort, J., O'Kane, D. J., Lee, J., and Carreira, L. A. (1983) Raman spectra of flavin bound in flavodoxins and in other flavoproteins. Evidence for structural variations in the flavinbinding region, Eur. J. Biochem. 131, 639-645.

40. Martin, C. B., Tsao, M. L., Hadad, C. M., and Platz, M. S. (2002) The reaction of triplet flavin with indole. A study of the cascade of reactive intermediates using density functional theory and timeresolved infrared spectroscopy, J. Am. Chem. Soc. 124, 72267234.

41. Hazekawa, I., Nishina, Y., Sato, K., Shichiri, M., Miura, R., and Shiga, K. (1997) A Raman study on the $\mathrm{C}(4)=\mathrm{O}$ stretching mode of flavins in flavoenzymes: hydrogen bonding at the $\mathrm{C}(4)=\mathrm{O}$ moiety, J. Biochem. (Tokyo) 121, 1147-1154.

42. Kim, M., and Carey, P. R. (1993) Observation of a carbonyl feature for riboflavin bound to riboflavin-binding protein in the red-excited Raman spectrum, J. Am. Chem. Soc. 115, 7015-7016.

43. Fahmy, K., Jager, F., Beck, M., Zvyaga, T. A., Sakmar, T. P., and Siebert, F. (1993) Protonation states of membrane-embedded carboxylic acid groups in rhodopsin and metarhodopsin II: a Fourier transform infrared spectroscopy study of site-directed mutants, Proc. Natl. Acad. Sci. U.S.A. 90, 10206-10210.

44. van Thor, J. J., Fisher, N., and Rich, P. R. (2005) Assignments of the Pfr-Pr FTIR difference spectrum of cyanobacterial phytochrome Cph1 using N-15 and C-13 isotopically labeled phycocyanobilin chromophore, J. Phys. Chem. B 109, 20597-20604.

45. Kay, C. W., Schleicher, E., Kuppig, A., Hofner, H., Rüdiger, W., Schleicher, M., Fischer, M., Bacher, A., Weber, S., and Richter, G. (2003) Blue light perception in plants. Detection and characterization of a light-induced neutral flavin radical in a C450A mutant of phototropin, J. Biol. Chem. 278, 10973-10982.

46. Tegoni, M., Gervais, M., and Desbois, A. (1997) Resonance Raman study on the oxidized and anionic semiquinone forms of flavocytochrome b2 and L-lactate monooxygenase. Influence of the structure and environment of the isoalloxazine ring on the flavin function, Biochemistry 36, 8932-8946.

47. Bowman, W. D., and Spiro, T. G. (1981) Normal mode analysis of lumiflavin and interpretation of resonance Raman spectra of flavoproteins, Biochemistry 20, 3313-3318.

48. Schleicher, E., Hessling, B., Illarionova, V., Bacher, A., Weber, S., Richter, G., and Gerwert, K. (2005) Light-induced reactions of Escherichia coli DNA photolyase monitored by Fourier transform infrared spectroscopy, FEBS J. 272, 1855-1866.

49. Benecky, M. J., Copeland, R. A., and Spiro, T. G. (1983) Resonance Raman spectra of flavin semiquinones stabilized by N5 methylation, Biochim. Biophys. Acta 760, 163-168.

50. Schelvis, J. P. M., Ramsey, M., Sokolova, O., Tavares, C., Cecala, C., Connell, K., Wagner, S., and Gindt, Y. M. (2003) Resonance Raman and $\mathrm{UV}-\mathrm{Vis}$ spectroscopic characterization of $\mathrm{FADH}^{\bullet}$ in the complex of photolyase with UV-damaged DNA, J. Phys. Chem. B 107, 12352-12362.

51. Kay, C. W., Feicht, R., Schulz, K., Sadewater, P., Sancar, A., Bacher, A., Möbius, K., Richter, G., and Weber, S. (1999) EPR, ENDOR, and TRIPLE resonance spectroscopy on the neutral flavin radical in Escherichia coli DNA photolyase, Biochemistry $38,16740-16748$

52. Jorns, M. S., Sancar, G. B., and Sancar, A. (1984) Identification of a neutral flavin radical and characterization of a second chromophore in Escherichia coli DNA photolyase, Biochemistry 23, 2673-2679.

53. Müller, F., Brüstlein, M., Hemmerich, P., Massey, V., and Walker, W. H. (1972) Light-absorption studies on neutral flavin radicals, Eur. J. Biochem. 25, 573-580.

54. Payne, G., and Sancar, A. (1990) Absolute action spectrum of E-FADH2 and E-FADH2-MTHF forms of Escherichia coli DNA photolyase, Biochemistry 29, 7715-7727.

55. Cashmore, A. R. (2003) Cryptochromes: enabling plants and animals to determine circadian time, Cell 114, 537-543.

56. Crosson, S., and Moffat, K. (2002) Photoexcited Structure of a Plant Photoreceptor Domain Reveals a Light-Driven Molecular Switch, Plant Cell 14, 1067-1075.

57. Fedorov, R., Schlichting, I., Hartmann, E., Domratcheva, T., Fuhrmann, M., and Hegemann, P. (2003) Crystal Structures and Molecular Mechanism of a Light-Induced Signaling Switch: The Phot-LOV1 Domain from Chlamydomonas reinhardtii, Biophys. J. 84, 2474-2482.

58. Harper, S. M., Neil, L. C., and Gardner, K. H. (2003) Structural basis of a phototropin light switch, Science 301, 1541-1544.

59. Harper, S. M., Christie, J. M., and Gardner, K. H. (2004) Disruption of the LOV-J $\alpha$ helix interaction activates phototropin kinase activity, Biochemistry 43, 16184-16192.

60. Jorns, M. S., Wang, B. Y., Jordan, S. P., and Chanderkar, L. P. (1990) Chromophore function and interaction in Escherichia coli DNA photolyase: reconstitution of the apoenzyme with pterin and/or flavin derivatives, Biochemistry 29, 552-561.

BI051964B 